\title{
Numerical simulation of the flow of a gas-liquid medium in a circular channel at cooling a high-temperature metal cylinder with a variable cross-section
}

\author{
Sergey Makarov ${ }^{1, *}$, Vyacheslav Dement'yev ${ }^{1, *}$, and Tatyana Makhneva ${ }^{1, *}$ \\ ${ }^{1}$ Institute of Mechanics of Ural Branch of the Russian Academy of Sciences, 34, T. Baramzinoy St., Izhevsk, 426067, Russia
}

\begin{abstract}
The numerical simulation results of the flow of a gas-liquid medium over the high-temperature metal cylinder surface in a circular channel are presented. The flow over the solid metal cylinder with a variable cross-section by a gas-liquid medium is considered. Based on the mathematical model taking into account the cooling-media-flow axial symmetry relative to the cylinder longitudinal axis, the results of the numerical simulation of the process of solving the two-dimensional nonstationary problem of the coupled heat exchange of the gas-liquid medium flow and metal cylinder are obtained. The control volume approach is used for solving the differential equation system. The flow field parameters are calculated using the algorithm SIMPLE. For the iterative solution of linear algebraic equations, Gauss-Seidel method with under-relaxation is used. The numerical calculation results are obtained for the flow hydrodynamic parameters of the gas-liquid medium at cooling the high-temperature metal cylinder by taking into account vaporization. The values of temperatures, flow velocities, and vapour distribution in the computational domain are determined. The variation intensity of the metal cylinder surface temperature depending on the gas-liquid flow velocity is analyzed.
\end{abstract}

\section{Introduction}

In metallurgy and engineering industry in the production of items from metals and alloys, the creation of rational conditions for cooling processed high-temperature metal bodies is an important problem in the procedures of strengthening of machine components. The selection of the heat-transfer properties of cooling media and methods and modes of their supply during cooling depend on the required cooling rate which is determined by the intensity of the heat transfer between a body and a medium. The formation of the required conditions of heat removal during the heat treatment of items from metals and alloys is paid much attention to. The works in this direction were conducted by N.V. Zimin, G.F. Golovin, D.B. Budrin, M.V. Kondratov, G.N. Kheyfets, A.C. Kadinova, Yu.G. Eysmond, V.G. Labeysh, K.Z. Shepelyakovskiy et al. The investigation results allowed to create a general pattern for the process of the cooling of heated metal workpieces at thermal treatment. However, practically in all the works, particular solutions and general conclusions are given only in a certain required range of cooling operation conditions for the standard set of properties of the workpiece material and cooling media. In this connection it is still important to conduct research studies in this direction.

The choice of the mode for cooling high-temperature metal workpieces mainly depends on the distribution of hydrodynamic and heat-transfer parameters of the liquid cooling flow.

The process of cooling of high-temperature metal bodies by a gas-liquid media flow is investigated under the leadership of A.M. Lipanov, Academician of RAN in the Institute of Mechanics of the Ural Branch of RAN. In Ref. [1], the numerical solution of the problem of cooling of a high-temperature solid metal cylinder by the air-water flow is presented, and the cooling liquid flows are considered quasi-stationary. In Ref. [2], the results of the mathematical simulation of cooling axisymmetric metal workpieces from structural alloyed steel by quasistationary flows of cooling liquid are given. The case of the flow over the high-temperature cylinder surface by a nonstationary one-dimensional water flow in the longitudinal axis direction is considered in Ref. [3]. In Ref. [4], the mathematical model of cooling a hollow cylindrical metal workpiece by longitudinal quasistationary water flows is considered. The algorithm of the numerical solution of the problem and the results of the numerical parametric calculations are given. The determination of the temperatures of the cooled cylinder was made by taking into account the time of the relaxation of thermal stresses and internal heat sources caused by polymorphic transformations in the material. The calculation results for the parameters of the heat exchange between the cylinder and medium flow depending on the geometry, thermophysical properties and the process time were obtained on the basis of the

\footnotetext{
* Corresponding author: ssmak15@mail.ru
} 
criterial dependences for the determination of the heattransfer conditions.

In Ref. [5], the mathematical model of the conjugate problem of the convective heat exchange at cooling a heated metal cylinder by the cooling-medium laminar flow is considered. Cooling occurs without liquid boiling. In Ref. [6], the mathematical model is offered for the coupled heat-exchange in the heterogeneous system 'solid body - gas-liquid medium' taking into account vaporization in the cooling longitudinal liquid flow by the surface of the high-temperature metal cylinder. The numerical algorithm was used for studying the cooling of the metal cylindrical workpiece from structural steel in Ref. [7].

The goal of the present work is to obtain the results of the numerical simulation of a gas-liquid flow in a circular channel at cooling a high-temperature metal cylinder with a variable cross-section and to investigate the variation of the temperature of the metal cylinder surface during cooling depending on the value of the gas-liquid flow velocity.

\section{Problem statement}

Let us consider the flow over a solid cylinder with a variable cross-section by a gas-liquid medium. The metal cylinder consists of two areas: the first of radius $r_{m 0}$ and length $L_{0}$ and the second of radius $r_{m}$ and length of $L-$ $L_{0}$. In the longitudinal direction of the horizontal axis $x$, the cylinder is flowed by the liquid flow with the initial velocity $u_{0}$. The thickness of the moving liquid is determined by the distance of the annular gap: on the area $L_{0}-$ from $r_{m 0}$ to $\eta$, and on the area $L-L_{0}-$ from $r_{m}$ to $\eta$. The thickness of the external ring is determined by the distance from $\eta$ to $r_{m l}$. Calculation points 1 and 2 are on the inner and outer boundary of the cylinder vertical surface, respectively, at $x=L_{0}$. The physical diagram of the computational domain is shown in Fig. 1.

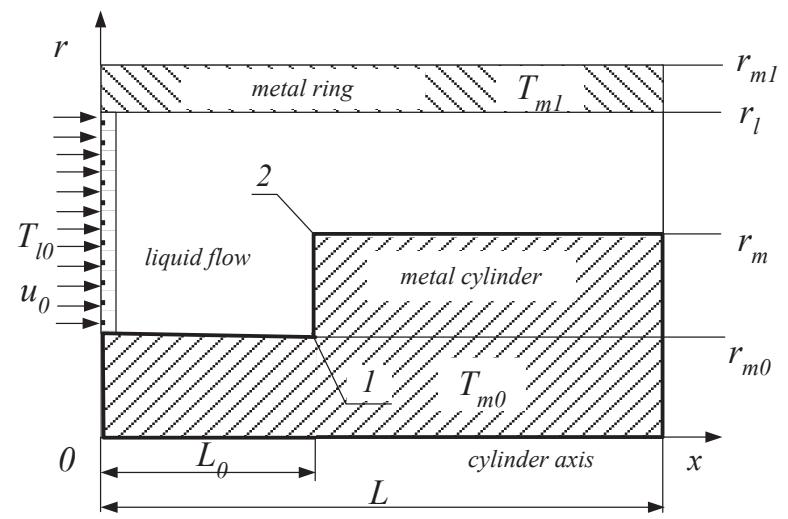

Fig.1. Physical diagram of the flow over the cylinderwith the variable cross-section

In the description of the process the following is assumed:

the liquid flow is considered axisymmetric relative to the longitudinal axis of the cylinder; the gravity forces action can be neglected;

the liquid flow is considered laminar; the calculation parameters in the direction $r$ vary continuously, and on the boundaries between the metal and liquid, the matching conditions in heat parameters are fulfilled;

by the cylinder surface, there is vaporization in the liquid flow;

the thermophysical parameters of the gas-liquid medium are determined by the relations describing the properties on the saturation line;

it is considered that the vapor density is much smaller than the liquid density and is described by the perfect gas equation;

the liquid vaporization is assumed to be equilibrium; the vapor pressure is equal to that of the liquid.

\section{Solution procedure}

The mathematical model of the coupled heat transfer of the two-dimensional non-stationary flow of the gasliquid medium and the metal cylinder taking into account the axial symmetry of the cooling medium flow relative to the longitudinal axis of the cylinder is considered in Refs. [6, 7].

The system of equations describing the gas-liquid medium flow is as follows:

$$
\begin{aligned}
& \rho \frac{\partial u}{\partial t}+\rho u \frac{\partial u}{\partial x}+\rho v \frac{\partial u}{\partial r}= \\
& =-\frac{\partial p}{\partial x}+\frac{\partial}{\partial x} \mu \frac{\partial u}{\partial x}+\frac{1}{r} \frac{\partial}{\partial r} r \mu \frac{\partial u}{\partial r} \\
& \rho \frac{\partial v}{\partial t}+\rho u \frac{\partial v}{\partial x}+\rho v \frac{\partial v}{\partial r}= \\
& =-\frac{\partial p}{\partial r}+\frac{\partial}{\partial x} \mu \frac{\partial v}{\partial x}+\frac{1}{r} \frac{\partial}{\partial r} r \mu \frac{\partial v}{\partial r}-\mu \frac{v}{r} \\
& \frac{\partial \rho}{\partial t}+\frac{\partial(\rho u)}{\partial x}+\frac{1}{r} \frac{\partial(r \rho v)}{\partial r}=0 \\
& \rho c \frac{\partial T}{\partial t}+\rho c u \frac{\partial T}{\partial x}+\rho c v \frac{\partial T}{\partial r}= \\
& =\frac{\partial}{\partial x} \lambda \frac{\partial T}{\partial x}+\frac{1}{r} \frac{\partial}{\partial r} r \lambda \frac{\partial T}{\partial r}-\dot{m}_{v} Q_{v} \\
& \rho \frac{\partial Y_{v}}{\partial t}+\rho u \frac{\partial Y_{v}}{\partial x}+\rho v \frac{\partial Y_{v}}{\partial r}=\dot{m}_{v}
\end{aligned}
$$

The specific mass velocity of the vaporization is found from the heat balance equation: $\dot{m}_{v}=\left(\rho c \Delta T^{*}\right) / Q_{v}$ where the reduced heat flux is determined by the following relation:

$$
\begin{gathered}
\Delta T^{*}=\left\{\begin{array}{l}
0, \text { если } T(t+\Delta t)<T_{s} \\
{\left[T(t+\Delta t)-T_{n}(t)\right] / \Delta t, \text { если } T(t+\Delta t)>T_{s}}
\end{array}\right. \\
T(t)=\max \left(T(t) ; T_{s}\right), \Delta t-\text { time increment, }
\end{gathered}
$$

$\boldsymbol{T}_{\boldsymbol{s}}$ - saturation temperature. 
The energy equation for the metal cylinder and the ring has the form:

$$
\rho_{m} c_{m} \frac{\partial T_{m}}{\partial t}=\frac{\partial}{\partial x} \lambda_{m} \frac{\partial T_{m}}{\partial x}+\frac{1}{r} \frac{\partial}{\partial r} r \lambda_{m} \frac{\partial T_{m}}{\partial r}
$$

The effective thermophysical parameters of the gasliquid medium are determined as follows:

$$
\begin{array}{cr}
\quad \Gamma=\Gamma_{1} Y_{v}+\left(1-Y_{v}\right) \Gamma_{2}, \quad \text { where } \quad \Gamma=\{\rho, \mu, \lambda\}, \\
\Gamma_{1}=\left\{\rho_{v}, \mu_{v}, \lambda_{v}\right\}, \\
c=\left(c_{v} \rho_{v} Y_{v}+c_{l} \rho_{l}\left(1-Y_{v}\right)\right) / \rho .
\end{array}
$$

At the calculation of the field of the gas-liquid medium flow, the values $\{\rho, \mu, \lambda\}$ are interpolated on the bounds of the control volume. The boundary conditions are taken as those in Refs. [6 - 8]. In the equations, the following symbols are used: $\boldsymbol{c}$ - specific heat capacity, $J /(\mathrm{kg} \cdot \mathrm{grad}) ; \rho$ - density, $\mathrm{kg} / \mathrm{m}^{3} ; \lambda$ - heat conduction, $W /(m \cdot g r a d) ; \boldsymbol{\mu}$ - dynamic viscosity, $P a \cdot s ; \boldsymbol{p}$-pressure, $P a$; $\boldsymbol{T}$ - temperature, $K ; \boldsymbol{t}$-time, $s ; \boldsymbol{r}$-radial coordinate, $m ; \boldsymbol{x}$ - longitudinal coordinate, $m ; \boldsymbol{L}$ - computational domain length, $m ; \boldsymbol{u}$ - velocity component along $x ; \boldsymbol{v}-$ velocity component along $r, \mathrm{~m} / \mathrm{s} ; \quad \boldsymbol{Y}$ - volume concentration, $\boldsymbol{Q}$ - specific heat of vaporization, $J / \mathrm{kg}$. Indices: $\boldsymbol{m}$ - metal, $\boldsymbol{l}$-liquid, $\boldsymbol{v}$-vapor, $\boldsymbol{\theta}$ - initial value, $\boldsymbol{s}$ - saturation parameters.

The system of differential equations (1) - (6) is solved by the control volume approach. The parameters of the flow field (1)-(3) are calculated using the algorithm SIMPLE [9]. For accelerating the iteration process convergence, the sequential bypass of the points of the computational grid in four directions is used [6]. For the calculations, the grid having a convergent profile on the boundaries 'metal cylinder-liquid' and 'liquidmetal ring' and the sizes of the boundary cells $10^{-5} \times 10^{-5}$ $\mathrm{m}$ is used at $r=r_{m}$ and $r=r_{l}$ for metal and liquid. As moving from the boundaries of the joint surfaces, the size of the grid cell increases according to the linear law with the coefficient 1.2. The computation step over the longitudinal coordinate $\mathrm{x}$ is constant, $10^{-4} \mathrm{~m}$.

\section{Results of the numerical calculations}

For the numerical calculation, let us take $r_{m 0}=0.005, r_{m}$ $=0.01 \mathrm{~m}, r_{l}=0.02 \mathrm{~m}, r_{m l}=0.03 \mathrm{~m}, L_{0}=0.01 \mathrm{~m}, L=$ $0.11 \mathrm{~m}, T_{m 0}=800^{\circ} \mathrm{C}, T_{l 0}=20^{\circ} \mathrm{C}$. The initial temperature of the metal ring is $T_{m l}=20^{\circ} \mathrm{C}$. The cylinder and ring are made out of steel 30 HGSN. The cooling liquid is water. The thermophysical parameters are taken according to Refs. $[10,11]$. The initial velocity of the liquid flow is $u_{0}=0.1 \mathrm{~m} / \mathrm{s}$. The computation time is $t=$ $1 \mathrm{~s}$. It is believed that at the initial time, in the circular channel the liquid medium has the initial velocity equal to the velocity of the flow.

Figures 2 and 4 show the values of the temperatures, flow velocity along the longitudinal coordinate, distribution of the vapor concentration in the computational domain at the gas-liquid flow over the high-temperature metal cylinder with the variable cross- section. The parameter values are given in the coordinates of the numbers of the grid cells in the longitudinal and radial directions.

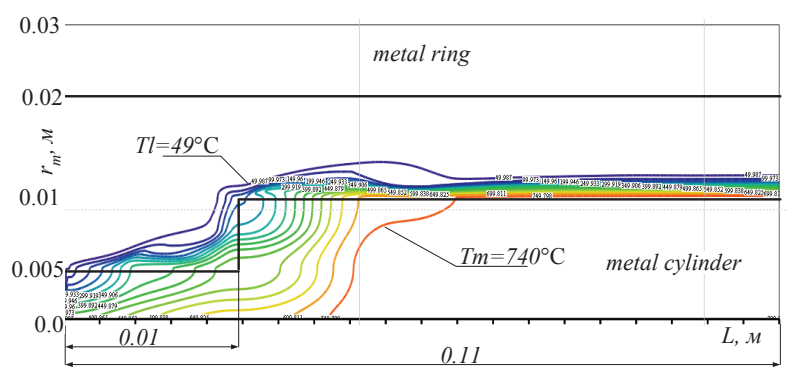

Fig.2. Temperature field

As can be seen from the conducted calculations, the temperature decrease of the cylinder from the boundaries of the gas-liquid medium contact in the direction of the axis takes place irregularly. Mostly the near-boundary regions situated perpendicularly to the liquid flow direction are cooled. The area $L-L_{0}$ of the surface of the cylinder of radius $r_{m}$ is cooled almost in two times more intensive than the other surface areas (Fig. 2). It is explained by the irregular distribution of the gas-liquid flow velocity and formation of the regions with lower velocities (Fig. 3). The decrease of the liquid flow velocity in the near-boundary regions leads to a significant overheating of the liquid which results in the intensive vaporization (Fig. 4) and increase of the of the metal cylinder surface temperature.

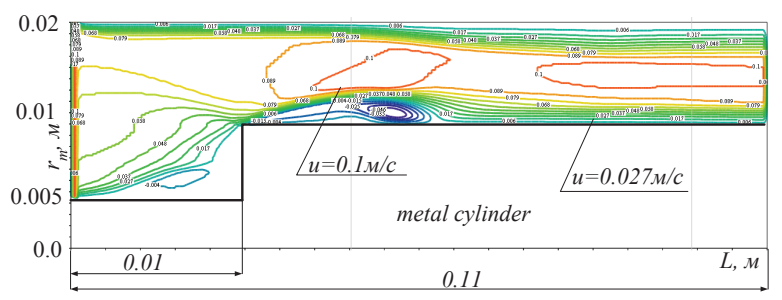

Fig.3. Field of the longitudinal velocity of the gas-liquid medium $u$

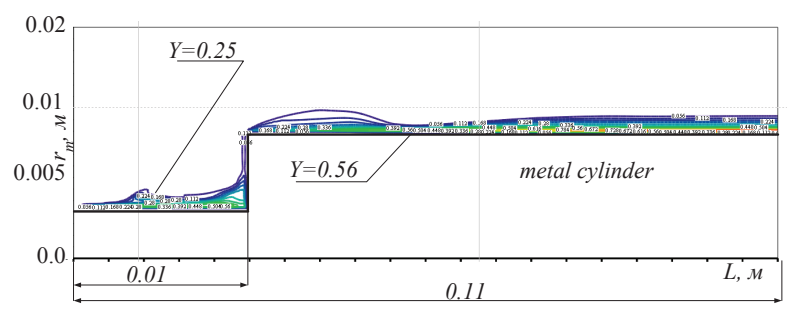

Fig.4. Field of the vapor concentration $Y$

Let us evaluate the influence of the gas-liquid medium flow velocity on the intensity of the metal cylinder surface cooling. The calculation will be conducted for two values of the initial velocity of the liquid flow $0.1 \mathrm{~m} / \mathrm{s}$ and $1 \mathrm{~m} / \mathrm{s}$. In Fig. 5, the variations of the temperature of the metal cylinder surface depending on the velocity of the gas-liquid medium flow are presented. 


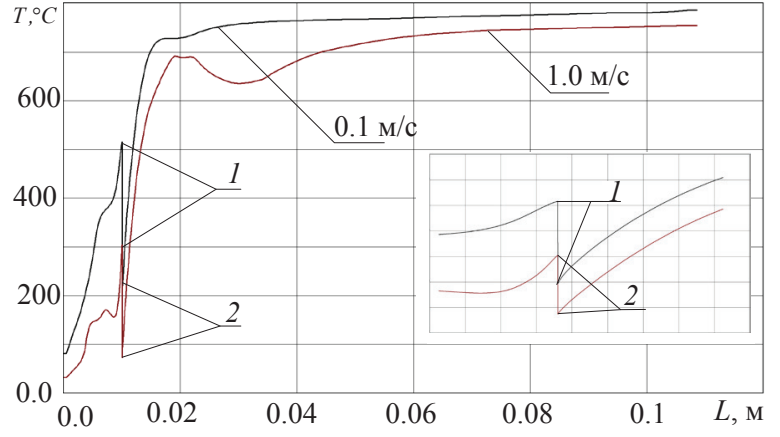

Fig.5. Metal cylinder surface temperature

At $x=L_{0}$, the surface temperature varies in the stepwise manner. The temperature at point 1 is higher than that at point 2 (see Fig. 1). For the flow velocity $0.1 \mathrm{~m} / \mathrm{s}$, at $x=L_{0}$, the range of the temperature variation between points 1 and 2 over the vertical cylinder surface is $(515-$ $192)^{\circ} \mathrm{C}$. At the flow velocity $1 \mathrm{~m} / \mathrm{s}$, it is $(305-73)^{\circ} \mathrm{C}$, respectively. It can be seen that with increasing velocity of the gas-liquid flow the intensity of cooling is increasing, and the surface of the cylinder with a larger radius is cooled more intensively. The results of the calculation show that for the equal time of calculation when the initial velocity of the cooling water flow increases tenfold, the temperature of the cylinder surface decreases on average by $50^{\circ} \mathrm{C}$ more than in the other case. The calculated values of the cylinder temperature confirm the regularities obtained at the conduction of natural experiments [12].

\section{Conclusion}

Using the mathematical model of the gas-liquid medium flow moving over the heated surface of the hightemperature metal cylinder with the variable crosssection, the numerical values of the temperature, gasliquid medium flow velocity and distribution of the vapor concentration in the computational domain have been obtained. The presented results of the numerical calculations have been obtained by taking into account the vaporization in the liquid. The variation of the temperature of the metal cylinder surface with respect to the time of the cooling process depending on the value of the medium flow velocity has been studied.

The work is sponsored by the Combined program of the Ural Branch of the Russian Academy of Sciences № 15-10-1-4.

\section{References}

1. A.M. Lipanov, S.S. Makarov, Mashinostroenie i inzhenernoe obrazovanie. 1 (2014)

2. S.S. Makarov, K.E. Chekmyshev, S.N. Khramov, E.V. Makarova, 3, 63 (2014)

3. S.S. Makarov, K.E. Chekmyshev, E.V. Makarova. Nauchno-tekhnicheskie vedomosti SPbGPU, 4, 207 (2014)

4. A.M. Lipanov, S.S. Makarov. Khimicheskaya fizika i mezoskopiya, 16, 4 (2014)
5. S.S. Makarov, A.I. Karpov, E.V. Makarova, Khimicheskaya fizika i mezoskopiya. 18, 1 (2016)

6. A.M. Lipanov, S.S. Makarov, A.I. Karpov, E.V. Makarova, Teplofizika i aeromekhanika. 24, 1 (2017) doi: 10.1134/S0869864317010061

7. S.S. Makarov, V.B. Dement'yev, E.V. Makarova, Procedia Engineering 150 (2016) doi: 10.1016/j.proeng. 2016.06.734

8. S.S. Makarov, Scientific and Technical Journal of Information Technologies, Mechanics and Optics 17, 2 (2017) doi: 10.17586/2226-1494-2017-17-2324-331.

9. S. Patankar, Numerical Heat Transfer and Fluid Flow (Press Roman, 1980)

10. M.P. Vukalovich. Thermophysical properties of water and vapor (M. Mashinostroenie, 1967).

11. V.G. Sorokin, M.A. Gervas'yev, Steels and alloys. Steel grade guide (M.: Intermet Inzhiniring, 2001)

12. V.G. Labeysh. Liquid cooling of high-temperature metal (L.: Izd-vo Leningr. un-ta, 1983) 